\title{
An alternative space-time meshless method for solving transient heat transfer problems with high discontinuous moving sources
}

\author{
Tonino Sophy ${ }^{a}$, Arthur Da Silva ${ }^{a}$, Ali Kribèche ${ }^{a}$, and Hamou Sadat ${ }^{b}$ \\ aDRIVE EA 1859, Université Bourgogne Franche Comté, Nevers, France; 'Institut P', Université de Poitiers, Centre \\ National de la Recherche Scientifique, Ecole Nationale Supérieure de Mécanique et Aérotechnique, Rue Pierre \\ Brousse, Bâtiment B25, TSA 41105, Poitiers Cedex 9, France
}

\begin{abstract}
The aim of this work is the development of a space-time diffuse approximation meshless method (DAM) to solve heat equations containing discontinuous sources. This work is devoted to transient heat transfer problems with static and moving heat sources applied on a metallic plate and whose power presents temporal discontinuities. The space-time DAM using classical weight function is convenient for continuous transient heat transfer. Nevertheless, for problems including discontinuities, some spurious oscillations for the temperature field occur. A new weight function, respecting the principle of causality, is used to eradicate the physically unexpected oscillations.
\end{abstract}

\section{Introduction}

Meshfree methods have become an attractive alternative for problems in computational engineering [1]. Several meshfree methods such as element-free Galerkin [2-4], meshless local Petrov-Galerkin [5], and moving least squares approximation have been proposed and these methods have achieved remarkable progress in solving a wide range of static and dynamic problems like heterogeneous heat conduction [4], wave propagation [6], or, recently, three-dimensional nonlinear wave equations [7]. Element-free Galerkin method has also been used to deal with moving heat source problems [8].

Usually, these dynamic problems are solved with a separated finite difference (FD) time scheme. Nevertheless, some problems, such as welding processes, dynamic deformation, or even fracture or moving boundary tracking, can be solved using a space-time discretization [9]. Space-time finite element meshes [10], like time-extended finite element method [11], have been used to simulate a thermal cycle [12] or to deal with thermoelastodynamic problems presenting some discontinuities [13], for fluid-structure interaction $[14,15]$ and, more recently, for time-dependent diffusion convection reaction problems, adding a module to COMSOL software [16]. Dumbser et al. [17] associated space-time grids with the finite volume method to solve nonlinear systems like Euler equations for compressive gas dynamics or magnetohydrodynamics problems. Space-time grids have also been used with finite difference-like methods to solve wave propagation problems [18, 19]. These methods are well known for going through a strong dependence on mesh properties.

As far as this note is concerned, it deals with space-time meshless methods that are over the past decade spread out such as the truly space-time meshfree collocation method developed by Netuzhylov et al. [20]. In their work, a time-slab stepping procedure complements a space-time meshfree collocation discretization. One of the advantages of a fully space-time discretization is that grid can be 


\section{Nomenclature}

\begin{tabular}{llll} 
A & calculation matrix & \multicolumn{2}{l}{ Greek symbols } \\
$\mathbf{B}$ & matrix system vector & $\boldsymbol{\alpha}$ & vector of coefficients \\
$B i$ & Biot number & $\alpha$ & thermal diffusivity \\
$c_{x}, c_{y}, c_{t}$ & distance coefficients & $\Delta x$ & dimensionless $x$ space step \\
$c_{p}$ & specific heat capacity & $\Delta y$ & dimensionless $y$ space step \\
$h$ & convection exchange coefficient & $\Delta \tau$ & dimensionless time step \\
$k$ & number of neighbors & $\theta$ & dimensionless temperature \\
$L_{r e f}$ & characteristic distance & $\lambda$ & thermal conductivity \\
$N$ & number of node & $\rho$ & density \\
$\mathbf{P}$ & polynomial basis function & $\sigma$ & weight function support radius \\
$P_{r e f}$ & reference power per unit volume & $\Phi$ & scalar field \\
$R_{c}$ & radius of influence using corrected distance. & $\omega$ & weight function \\
$r$ & dimensionless distance & $\Omega$ & domain \\
$S$ & dimensionless heat source & & \\
$t$ & real time & Subscripts \\
$T$ & temperature in ${ }^{\circ} \mathrm{C}$ & $c$ & corrected \\
$T_{r e f}$ & reference temperature & $i, j$ & node index \\
$x, y, \tau$ & dimensionless space-time coordinates & ext & external \\
$x_{i}, y_{i}, \tau_{i}$ & dimensionless distance between calculation node & end & end of time calculation \\
$\mathbf{X}$ & and neighboring node & init & initial \\
& node location vector & $M$ & point
\end{tabular}

independently refined in time and/or space which can be suitable in case of moving boundaries, complex shapes, and crack growth. It also improves an accuracy made by Dumbser et al. [17].

The main purpose of this note is to propose a fully space-time meshless discretization method avoiding any secondary finite difference time-stepping procedure. It is shown that this method is convenient for continuous transient conductive or radiative [21] heat transfer problems. But, while solving partial differential equation containing strong temporal discontinuities, spurious numerical oscillations occur. Although refinement has been carried out to attempt to remove these oscillations, they still remained. One solution found to eradicate these unexpected oscillations was the use of an alternative weight function. This methodology has already been presented and tested in a previous note [22] when solving $1 \mathrm{D}$ spatial problems concerning the conduction in a bar with an internal fixed heat source depending on time. In this present paper, the calculation is extended to a $2 \mathrm{D}$ spatial problem to test the robustness of the method at higher dimension. Furthermore, instead of being restricted to a static heat source whose power discontinuously changes against time, the case of a moving heat source is also tested.

In Section 2, a succinct mathematical formulation of the space-time diffuse approximation method is given. Section 3 presents the classical weight function. Then, Section 4 deals with a modified weight function suitable in the presence of highly discontinuous sources. Section 5 gives three different numerical examples to test the robustness of the modified weight function.

\section{Mathematical formulation}

In the proposed approach, the space-time domain is first discretized into a set of $N$ points $M_{i}$. If we consider a scalar field $\Phi$ and its discrete values $\Phi_{i}$ corresponding to the $N$ nodes, the meshless method allows estimating derivatives (up to some order) of $\Phi$ at the $N$ nodes by minimizing a quadratic error. The Taylor expansion of $\Phi$ at the calculation node is truncated at the second order by writing:

$$
\Phi_{M_{i}}^{\text {estimated }}=\left\langle\mathbf{P}\left(\mathbf{X}_{M_{i}}-\mathbf{X}_{M}\right)\right\rangle\left\langle\alpha\left(\mathbf{X}_{M}\right)\right\rangle^{T}
$$


where $\left\langle\mathbf{P}\left(\mathbf{X}_{M_{i}}-\mathbf{X}_{M}\right)\right\rangle$ is the space-time polynomial basis function vector and $\left\langle\alpha\left(\mathbf{X}_{M}\right)\right\rangle^{T}$ is a column vector containing the partial derivatives:

$$
\left\langle\mathbf{P}\left(\mathbf{X}_{M_{i}}-\mathbf{X}_{M}\right)\right\rangle=\left\langle 1, x_{i}, y_{i}, \tau_{i}, x_{i}^{2}, y_{i}^{2}, \tau_{i}^{2}, x_{i} \cdot y_{i}, x_{i} \cdot \tau_{i}, y_{i} \cdot \tau_{i}\right\rangle
$$

with $x_{i}=X_{M_{i}}-X_{M} ; y_{i}=Y_{M_{i}}-Y_{M} ; \tau_{i}=\tau_{M_{i}}-\tau_{M}$

$$
\left\langle\alpha\left(\mathbf{X}_{M}\right)\right\rangle^{T}=\left\langle\Phi, \frac{\partial \Phi}{\partial x}, \frac{\partial \Phi}{\partial y}, \frac{\partial \Phi}{\partial \tau}, \frac{1}{2 !} \frac{\partial^{2} \Phi}{\partial x^{2}}, \frac{1}{2 !} \frac{\partial^{2} \Phi}{\partial y^{2}}, \frac{\partial^{2} \Phi}{\partial \tau^{2}}, \frac{\partial^{2} \Phi}{\partial x \partial y}, \frac{\partial^{2} \Phi}{\partial x \partial \tau}, \frac{\partial^{2} \Phi}{\partial y \partial \tau}\right\rangle^{T}
$$

The coefficients of vector $\boldsymbol{\alpha}$ are determined by minimizing the following quadratic form:

$$
I\left(\alpha_{M}\right)=\sum_{j=1}^{n}\left\{\omega\left(M_{j}, M\right) \cdot\left[\varphi_{j}-\left\langle p\left(M_{j}, M\right)\right\rangle \cdot\left\langle\alpha_{M}\right\rangle^{T}\right]^{2}\right\}
$$

where $\omega\left(M_{j}, M\right)$ is a continuous positive weighting function which is maximum at $M$ and decreases quickly.

It can therefore be written for each value of $i$ :

$$
\frac{\partial I\left(\alpha_{M}\right)}{\partial \alpha_{i}}=0
$$

This minimization process leads to the following matrix system:

$$
\left[\mathbf{A}\left(\mathbf{X}_{M}\right)\right]\left\langle\boldsymbol{\alpha}\left(\mathbf{X}_{M}\right)\right\rangle^{T}=\left\langle\mathbf{B}\left(\mathbf{X}_{M}\right)\right\rangle^{T}
$$

where the square matrix $\mathbf{A}\left(\mathbf{X}_{M}\right)$ and the column vector $\mathbf{B}\left(\mathbf{X}_{M}\right)$ are, respectively, defined as:

$$
\begin{gathered}
{\left[\mathbf{A}\left(\mathbf{X}_{M}\right)\right]=\sum_{i=1}^{N} \omega\left(\mathbf{X}_{M}, \mathbf{X}_{M_{i}}-\mathbf{X}_{M}\right)\left\langle\mathbf{P}\left(\mathbf{X}_{M_{i}}-\mathbf{X}_{M}\right)\right\rangle^{T}} \\
\left\langle\mathbf{B}\left(\mathbf{X}_{M}\right)\right\rangle^{T}=\sum_{i=1}^{N} \omega\left(\mathbf{X}_{M}, \mathbf{X}_{M_{i}}-\mathbf{X}_{M}\right)\left\langle\mathbf{P}\left(\mathbf{X}_{M_{i}}-\mathbf{X}_{M}\right)\right\rangle^{T} \Phi_{i}
\end{gathered}
$$

The approximation matrix $\mathbf{A}\left(\mathbf{X}_{M}\right)$ has the following form:

$$
\begin{gathered}
{\left[\mathbf{A}\left(\mathrm{X}_{M}\right)\right]=\sum_{i=1}^{N} \omega\left(\mathbf{X}_{M}, \mathbf{X}_{M_{i}}-\mathbf{X}_{M}\right)} \\
{\left[\begin{array}{cccccccccc}
1 & x_{i} & y_{i} & \tau_{i} & x_{i}^{2} & y_{i}^{2} & \tau_{i}^{2} & x_{i} y_{i} & x_{i} \tau_{i} & y_{i} \tau_{i} \\
* & x_{i}^{2} & x_{i} y_{i} & x_{i} \tau_{i} & x_{i}^{3} & x_{i} y_{i}^{2} & x_{i} \tau_{i}^{2} & x_{i}^{2} y_{i} & x_{i}^{2} \tau_{i} & x_{i} y_{i} \tau_{i} \\
* & * & y_{i}^{2} & y_{i} \tau_{i} & y_{i} x_{i}^{2} & y_{i}^{3} & y_{i} \tau_{i}^{2} & x_{i} y_{i}^{2} & x_{i} y_{i} \tau_{i} & y_{i}^{2} \tau_{i} \\
* & * & * & \tau_{i}^{2} & x_{i}^{2} \tau_{i} & y_{i}^{2} \tau_{i} & \tau_{i}^{3} & x_{i} y_{i} \tau_{i} & x_{i} \tau_{i}^{2} & y_{i} \tau_{i}^{2} \\
* & * & * & * & x_{i}^{4} & x_{i}^{2} y_{i}^{2} & x_{i}^{2} \tau_{i}^{2} & x_{i}^{2} y_{i} & x_{i}^{3} \tau_{i} & x_{i}^{2} y_{i} \tau_{i} \\
* & * & * & * & * & y_{i}^{4} & y_{i}^{2} \tau_{i}^{2} & x_{i} y_{i}^{3} & x_{i} y_{i}^{2} \tau_{i} & y_{i}^{3} \tau_{i} \\
* & * & * & * & * & * & \tau_{i}^{4} & x_{i} \tau_{i}^{2} y_{i} & x_{i} \tau_{i}^{3} & y_{i} \tau_{i}^{3} \\
* & * & * & * & * & * & * & x_{i}^{2} y_{i}^{2} & x_{i}^{2} y_{i} \tau_{i} & x_{i}^{2} y_{i}^{2} \tau_{i} \\
* & * & * & * & * & * & * & * & x_{i}^{2} \tau_{i}^{2} & x_{i} y_{i} \tau_{i}^{2} \\
* & * & * & * & * & * & * & * & * & y_{i}^{2} \tau_{i}^{2}
\end{array}\right]}
\end{gathered}
$$


Thus, $\mathbf{A}\left(\mathbf{X}_{M}\right)$ is a sum of $k$ rank 1 matrices. To avoid $\mathbf{A}\left(\mathbf{X}_{M}\right)$ being singular, $k$ has to be at least equal to $\boldsymbol{\alpha}\left(\mathbf{X}_{M}\right)$ 's dimension. The inversion of system [Eq. (6)] leads to the expression of $\boldsymbol{\alpha}\left(\mathbf{X}_{M}\right)$ components which are the derivatives of $\Phi$ at the calculation node in terms of the scalar value $\Phi_{i}$ at each neighboring node:

$$
\left\{\begin{array}{c}
\Phi \\
\partial \Phi / \partial x \\
\partial \Phi / \partial y \\
\partial \Phi / \partial \tau \\
\frac{1}{2 !} \partial^{2} \Phi / \partial x^{2} \\
\frac{1}{2 !} \partial^{2} \Phi / \partial y^{2} \\
\frac{1}{2 !} \partial^{2} \Phi / \partial \tau^{2} \\
\partial^{2} \Phi / \partial x \partial y \\
\partial^{2} \Phi / \partial x \partial \tau \\
\partial^{2} \Phi / \partial y \partial \tau
\end{array}\right\}=\left[\mathbf{A}\left(\mathbf{X}_{M}\right)\right]^{-1} \cdot \sum_{i=1}^{N} \omega\left(\mathbf{X}_{M}, \mathbf{X}_{M_{i}}-\mathbf{X}_{M}\right) \cdot\left\{\begin{array}{c}
1 \\
x_{i} \\
y_{i} \\
\tau_{i} \\
x_{i}^{2} \\
y_{i}^{2} \\
\tau_{i}^{2} \\
x_{i} \cdot y_{i} \\
x_{i} \cdot \tau_{i} \\
y_{i} \cdot \tau_{i}
\end{array}\right\} \cdot \Phi_{i}
$$

In that case, a partial derivative equation (PDE) being an expression involving some derivatives contained in $\boldsymbol{\alpha}\left(\mathbf{X}_{M}\right)$ will be replaced at each calculation node by a weighted linear combination of the $\Phi_{\mathrm{i}}$ corresponding to some selected neighboring nodes. Hence, transcribing the PDE on the entire domain (at all the calculation nodes) will lead to a matrix system, whose unknowns will be the different values of the field at the different nodes. This matrix system will be solved by an inversion procedure or, and that will be our case, by an ILUT(k) preconditioned BiCGStab solver.

Boundary conditions can be easily set by replacing the line corresponding to a boundary node by the meshless approximation of the imposed condition. Concerning Dirichlet boundary conditions, two approaches can be used. The first consists in, as previously mentioned, using the first line of the inverse matrix $\mathbf{A}^{-1}\left(\mathbf{X}_{M}\right)$ for the approximation of the field. This approach is still an approximation of the field but allows doing interpolations. The second one is the diagonal unity method. It has the advantage to set the exact value for the boundary condition.

\section{Classical weight function}

In this approximation, a weight function $\omega$ is involved. We have chosen the following Gaussian function:

$$
\begin{aligned}
& \left.\omega\left(\mathbf{X}_{M}, \mathbf{X}_{M_{i}}-\mathbf{X}_{M}\right)=\exp \left[-3 \ln (10) \cdot \frac{\left\|\mathbf{X}_{M_{i}}-\mathbf{X}_{M}\right\|^{2}}{\left[\sigma\left(\mathbf{X}_{M}\right)\right]^{2}}\right)\right] \\
& \omega\left(\mathbf{X}_{M}, \mathbf{X}_{M_{i}}-\mathbf{X}_{M}\right)=0 \text { if }\left\|\mathbf{X}_{M_{i}}-\mathbf{X}_{M}\right\|^{2} \geq\left[\sigma\left(\mathbf{X}_{M}\right)\right]^{2}
\end{aligned}
$$

where $\sigma\left(\mathbf{X}_{M}\right)$ is the weight function support radius. In the classical approach, the definition of the weight function will impose a node selection area outside which $\omega=0$.

Thus, sums including the entire domain nodes must be considered as sums truncated to the only $\mathrm{k}$ nodes belonging to the vicinity of the calculation node $\mathbf{X}_{M}$. A minimum of 27 nodes is recommended to avoid matrix $\mathrm{A}\left(\mathbf{X}_{M}\right)$ from being singular and to consider all space-time directions [23].

In the neighbors research process, if the dimensionless space and time steps verify $\Delta x=\Delta y=\Delta \tau$, the influence zone delimited by the weight function's support is a sphere of radius $R\left(\mathbf{X}_{M}\right)$. This radius may be altered regarding node's position. If the condition $(\Delta x=\Delta y=\Delta \tau)$ is not verified, the influence zone is no longer circular but ellipsoidal. 
A corrected norm is introduced as in Eq. (12):

$$
\|\|_{c}: \begin{gathered}
\Omega \rightarrow \Re \\
\left(\mathbf{X}_{M_{i}}, \mathbf{X}_{M}\right) \rightarrow r_{\mathrm{c}}
\end{gathered}
$$

where $r_{\mathrm{c}}$ is a $\Re^{3} \rightarrow \Re$ application describing the following distance (Eq. 13):

$$
r_{\mathrm{c}}=\sqrt{\left(\frac{x_{i}}{c_{x}}\right)^{2}+\left(\frac{y_{i}}{c_{y}}\right)^{2}+\left(\frac{\tau_{i}}{c_{\tau}}\right)^{2}}
$$

where $c_{x}, c_{y}$, and $c_{\tau}$ are coefficients verifying the following conditions:

$$
c_{\alpha}=\frac{\min _{\Omega}\left(\Delta_{\alpha}\right)}{\min _{\beta \in\{x, y, \tau\}}\left(\min _{\Omega}(\Delta \beta)\right)} \text { for } \alpha=x, y, \text { or } \tau
$$

Then, using this corrected norm, the influence zone turns to be an ellipsoid which fits with the weight function support (Eq.(14)):

$$
\sigma\left(\mathrm{X}_{M}\right)=R_{\mathrm{c}}\left(\mathrm{X}_{M}\right)
$$

Furthermore, the term

$$
\left\|\mathbf{X}_{M_{i}}-\mathbf{X}_{M}\right\| \text { in (Eq.(11)) is replaced by }\left\|\mathbf{X}_{M_{i}}-\mathbf{X}_{M}\right\|_{\mathrm{c}} \text { [Eq.(12)]. }
$$

An iterative procedure is applied until the minimum of 27 neighboring nodes is reached.

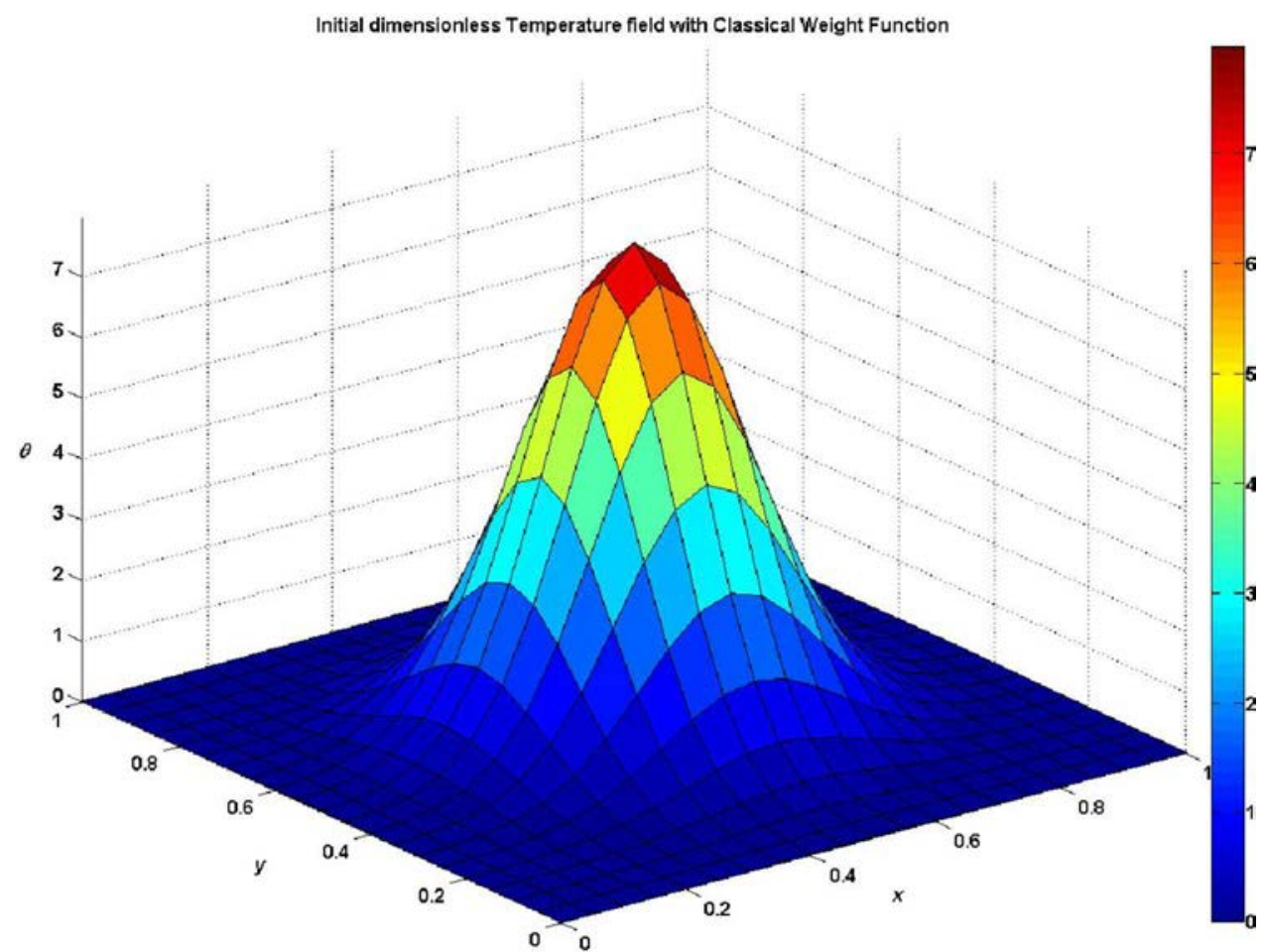

Figure 1. Initial dimensionless temperature field on a metallic plate at $\tau_{\text {init }}=0.01$. 


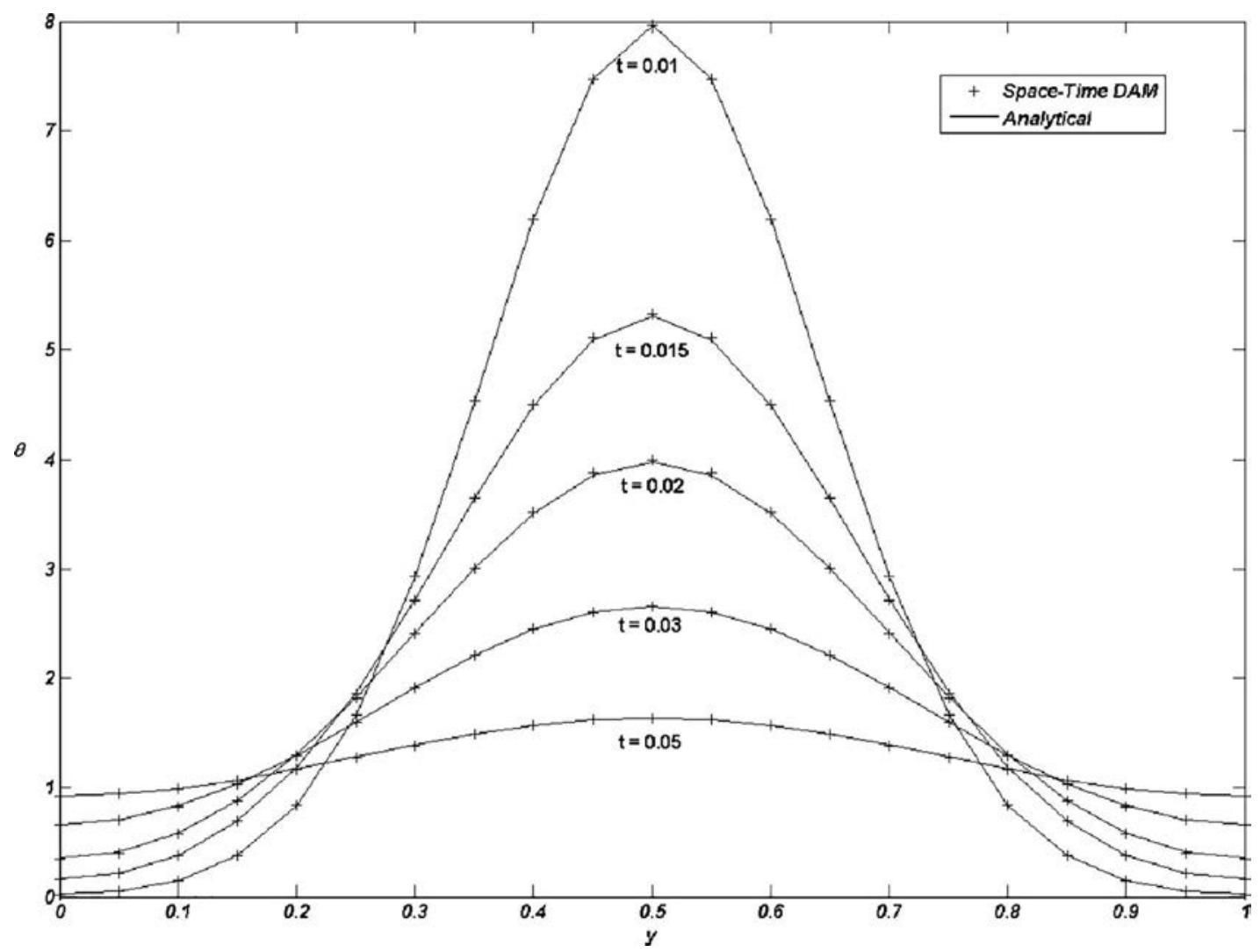

Figure 2. Dimensionless temperature distribution at $x=0.5$, for $\tau=0.01,0.015,0.02,0.03$, and 0.05 .

Table 1. Relative errors for different grids, $n_{x}, n_{y}$, and $n_{\tau}$ are the number of nodes, respectively, in $x, y$, and $\tau$ directions.

\begin{tabular}{lccc}
\hline Grid $n_{x} \times n_{y} \times n_{\mathrm{T}}$ & $11 \times 11 \times 21$ & $21 \times 21 \times 21$ & $21 \times 21 \times 41$ \\
$e_{\mathrm{r}}(\%)$ & 1.35 & 0.49 & 0.18 \\
\hline
\end{tabular}

\section{Use of a "backward" modified weight function}

The space-time meshless approach described so far has proven to work well in 1D spatial heat conduction problems as long as the source term if any is sufficiently smooth [22]. Otherwise, numerical oscillations take place in the solution procedure when highly discontinuous sources are considered. This is due to the fact that the weight function used in the formulation may select some nodes at future times. We have therefore proposed to choose a modified weight function which uses only nodes at current and previous times. We shall refer to this weight function as to a "backward" weight function whose main characteristics in $2 \mathrm{D}$ spatial problems are now briefly presented. In the classical

Table 2. Physical properties of the $[0.1 \mathrm{~m} \times 0.1 \mathrm{~m}]$ metallic plate (mild steel) and initial and external temperatures.

\begin{tabular}{lc}
\hline Parameters & Value \\
\hline$L_{\text {ref }}$ & $0.1 \mathrm{~m}$ \\
$\lambda$ & $46 \mathrm{~W} / \mathrm{m} / \mathrm{K}$ \\
$c_{\mathrm{p}}$ & $500 \mathrm{~J} / \mathrm{kg}^{1} / \mathrm{K}$ \\
$\rho$ & $7830 \mathrm{~kg} / \mathrm{m}$ \\
$T_{\text {init }}$ & $20^{\circ} \mathrm{C}$ \\
$T_{\text {ext }}$ & $15^{\circ} \mathrm{C}$ \\
\hline
\end{tabular}


approach, the only condition advocated for node selection, in the space-time $(x, y, \tau)$ domain, is a minimum of 27 nodes inside the discriminative radius $R_{\mathrm{c}}\left(\boldsymbol{X}_{M}\right)$. In the modified "backward" weight function approach, an additional condition restricts the neighbors to those located at times lower than the calculation node's time. Selection process will then reach neighbors farther than those of the classical approach. If space and time steps are equal, the neighboring zone is a "south" hemisphere, otherwise it is a semiellipsoid.

Although corrected distance (Eq. (13)) is still applied in the new selection process, it is avoided for the calculation of the weight function value. It means that the term $\left\|\mathbf{X}_{M_{i}}-\mathbf{X}_{M}\right\|$ in (Eq.(11)) is no longer replaced by $\left\|\mathbf{X}_{M_{i}}-\mathbf{X}_{M}\right\|_{c}$ [Eq.(12)]. Furthermore, the weight function's support radius $\sigma\left(X_{M}\right)$ is no longer equal to the radius of influence $R_{c}\left(X_{M}\right)$. In the new approach, the weight function support radius is fixed to an unvarying value $\sigma^{*}=1.1 \cdot \max [\Delta x, \max (\Delta y, \Delta \tau)]$. The discussion of the chosen coefficient 1.1 has already been made in [22].

\section{Numerical examples}

This section is devoted to the presentation of numerical results obtained by solving the heat equation with the associated boundary conditions which read:

$$
\frac{\partial \theta}{\partial \tau}-\left(\frac{\partial^{2} \theta}{\partial x^{2}}+\frac{\partial^{2} \theta}{\partial x^{2}}\right)=S(x, y, \tau)
$$

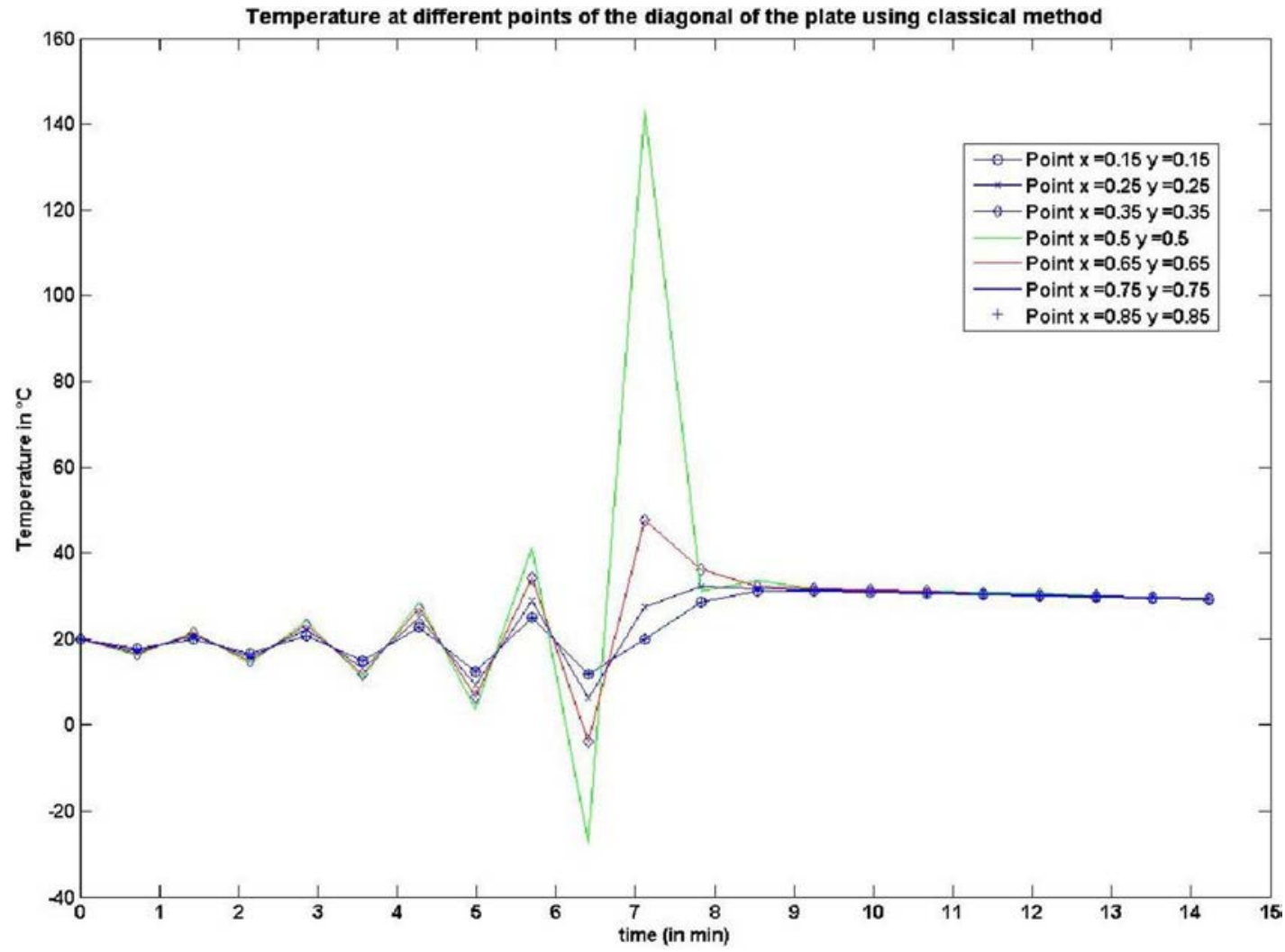

Figure 3. Temperature against time along the diagonal of the plate for a time bounded Gaussian static heat source of $7.5 \mathrm{~mm}$ standard deviation applied on the middle of a flat steel plate, at middle time, during $t_{\text {heat }}=43 \mathrm{~s}$, for $n_{x}=n_{y}=n_{\mathrm{\tau}}=21, \mathrm{Bi}=0.1$, and $P_{\max }=100 \mathrm{MW} / \mathrm{m}^{3}$ (using classical method). 


$$
\begin{aligned}
& \text { at } x=0: \frac{\partial \theta}{\partial x}=+B i \cdot \theta \text { at } x=1: \frac{\partial \theta}{\partial x}=-B i \cdot \theta, \\
& \text { at } y=0: \frac{\partial \theta}{\partial x}=+B i \cdot \theta \text { at } y=1: \frac{\partial \theta}{\partial y}=-B i \cdot \theta
\end{aligned}
$$

The dimensionless temperature is defined as $\theta=\frac{T-T_{\text {ext }}}{T_{\text {init }}-T_{\text {ext }}}$, where $T$ is the material temperature at time $\tau, T_{\text {init }}$ is the initial temperature, and $T_{\text {ext }}$ is the external temperature. $S=\frac{P}{P_{\text {ref }}}$ is the dimensionless volumetric source where the reference source power is $P_{\text {ref }}=\frac{\lambda\left(T_{\text {init }}-T_{\text {ext }}\right)}{L_{\text {ref }}^{2}}$. Finally, Bi is the classical Biot number.

\subsection{First test: Transient diffusion in a metallic plate}

We consider here the problem of laser pulse heating of a sheet of metal solved in [24] and whose analytical solution [25] is:

$$
\left.\theta(x, y, \tau)=\sum_{n=-\infty}^{+\infty} \frac{1}{2 \sqrt{\pi \tau}} e^{-\frac{(x+n-0.5)^{2}}{4 \tau}}\right)\left(\sum_{n=-\infty}^{+\infty} \frac{1}{2 \sqrt{\pi \tau}} e^{-\frac{(y+n-0.5)^{2}}{4 \tau}}\right)
$$

This solution corresponds to the response to a unit heat pulse deposited into the center of a unit side square domain with adiabatic boundary conditions. As in [24], we solve here the heat equation with no source term and with $\mathrm{Bi}=0$, but we start the calculations at a dimensionless time: $\tau=\tau_{\text {init }}$ $=0.01$ at which temperature is given by the analytical solution [Eq. (16)]: $\theta=\theta_{\text {init }}\left(x, y, \tau_{\text {init }}\right)$ presented

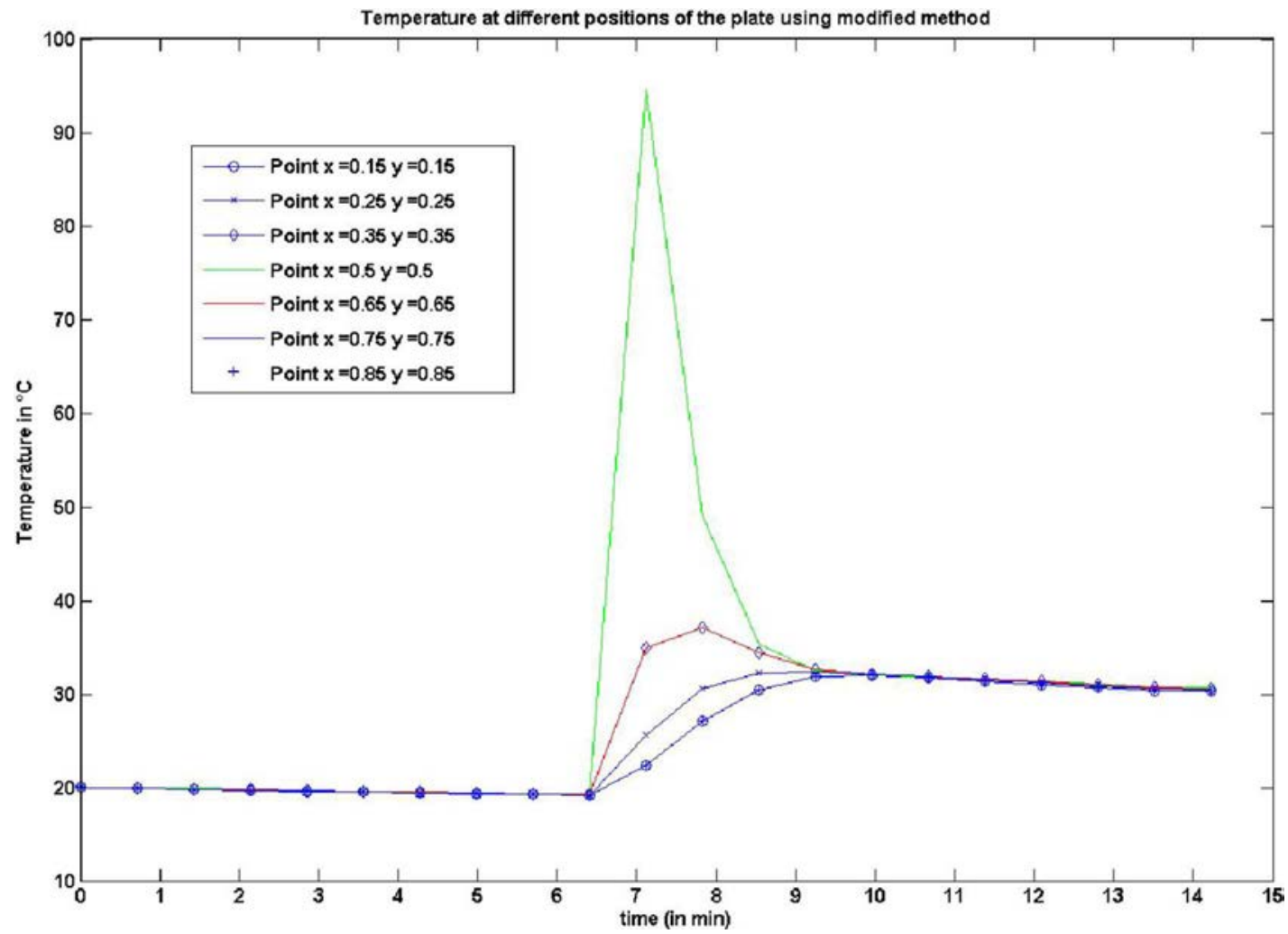

Figure 4. Temperature against time for a static Gaussian heat source of $7.5 \mathrm{~mm}$ standard deviation applied on the middle of a flat steel plate, at middle time, during $t_{\text {heat }}=43 \mathrm{~s}$, for $n_{x}=n_{y}=n_{\tau}=21, B i=0.1$, and $P_{\max }=100 \mathrm{MW} / \mathrm{m}^{3}$ (using modified method). 
in Figure 1. The whole calculation domain in our space-time representation is therefore $(x, y, \tau) \in$ $[0,1]^{2} \times[0.01,1]$.

Figure 2 compares analytical and calculated temperature obtained with the classical weight function for several times at $x=0.1$. A very good agreement is observed. As expected, when time tends to infinity, the temperature distribution becomes uniform over the whole plate. Table 1 shows that the relative error $e_{\mathrm{r}}=\frac{\theta_{\text {rms }}}{\theta_{\text {analytical }}}$, where $\theta_{\text {rms }}=\sqrt{\sqrt{\left(\theta^{\text {DAM }}-\theta^{\text {analytical }}\right)^{2}}}$, decreases from $1.35 \%$ for a $11 \times 11 \times 21$ grid to $0.18 \%$ for a $21 \times 21 \times 41$ grid.

As the $21 \times 21 \times 21$ grid leads to an error lower than $1 \%$, the next simulations will be conducted with this discretization.

\subsection{Second test: Transient heat conduction with a discontinuous time-dependent heat source}

We now consider the heat conduction problem involving a discontinuous time-dependent heat source term $S(x, y, \tau)$ given as follows:

$$
S(x, y, \tau)=\left\{\begin{array}{l}
\frac{P_{\max }}{P_{\text {ref }}} \cdot e^{\left(-r^{2} / \sigma_{\text {source }}^{2}\right)} \text { if }\left(\tau=0.5 \text { and }\left((x-\tau)^{2}+(y-\tau)^{2}\right) \leq \sigma_{\text {source }}^{2}\right) \\
0 \text { else }
\end{array}\right.
$$

where $r=\sqrt{(x-\tau)^{2}+(y-\tau)^{2}}$ and $\sigma_{\text {source }}$ is the standard deviation of the bell curve $\left(\sigma_{\text {source }}=1.5 \times \Delta x\right)$. The source heats the plate during a dimensionless time step $\Delta \tau$. $\tau_{\text {end }}$ is the dimensionless final time of the

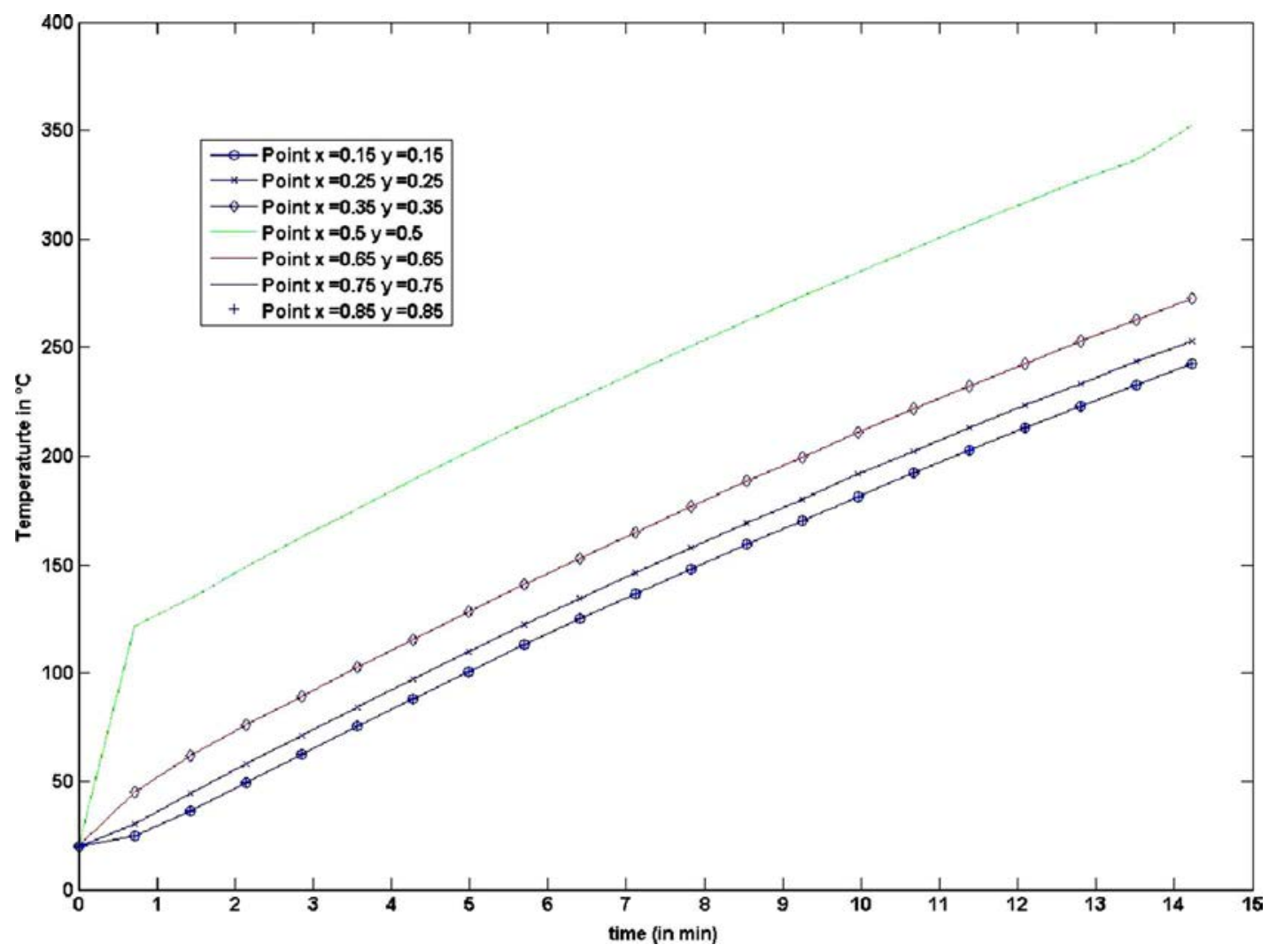

Figure 5. Temperature against time along the diagonal of the plate for a Gaussian constant heat source of $7.5 \mathrm{~mm}$ standard deviation applied on the middle of a flat steel plate, during $t_{\text {heat }}=t_{\text {end }}=14 \min 13 \mathrm{~s}$, for $n_{x}=n_{y}=n_{\mathrm{\tau}}=21, \mathrm{Bi}=0.1$, and $P_{\max }=100 \mathrm{MW} / \mathrm{m}^{3}$. 
problem set to 1 corresponding to a real time $t_{\mathrm{end}}=14 \mathrm{~min} 13 \mathrm{~s}$. A Biot number $\mathrm{Bi}=0.1$ corresponding to forced convection in air was chosen and the volumetric power source $P_{\max }$ was set to $100 \mathrm{MW} / \mathrm{m}^{3}$. The material properties are given in Table 2 and the problem was solved on a $21 \times 21 \times 21$ grid.

As shown in Figure 3, spurious oscillations appear in the temperature profiles when using the classical weight function. This could be explained by the fact that the principle of causality is not respected, especially in case of high discontinuities. Indeed, some calculation nodes are related to future neighboring nodes. To get rid from these oscillations, the use of the "backward" weight function is necessary. Figure 4 shows that the results are then completely smooth and correct.

It is worth noting that these oscillations are not present in the case of a smooth continuous source term when using the classical weight function. As a matter of example, we show in Figure 5 the temperature evolution at several points when applying the following Gaussian source applied continuously during all the process:

$$
S(x, y, \tau)=\frac{P_{\max }}{P_{\text {ref }}} \cdot e^{\left(-r^{2} / \sigma_{\text {source }}^{2}\right)} \text { if }\left((x-0.5)^{2}+(y-0.5)^{2}\right) \leq \sigma_{\text {source }}^{2}, \quad \forall \tau
$$

\subsection{Third test: Transient heat conduction with a moving heat source}

We finally present the results obtained when the following moving heat source starting at dimensionless space time $(x, y, \tau)=(0.5,0.5,05)$ is applied:

$$
S(x, y, \tau)=\left\{\begin{array}{l}
\frac{P_{\max }}{P_{\text {ref }}} \cdot e^{\left(-r^{2} / \sigma_{\text {source }}^{2}\right)} \cdot e^{\left[-\frac{(r-0.5)^{2}}{0.2^{2}}\right]} \text { if }\left(\tau \geq 0.5 \text { and }\left((x-\tau)^{2}+(y-\tau)^{2}\right) \leq \sigma_{\text {source }}^{2}\right) \\
0 \text { else }
\end{array}\right.
$$

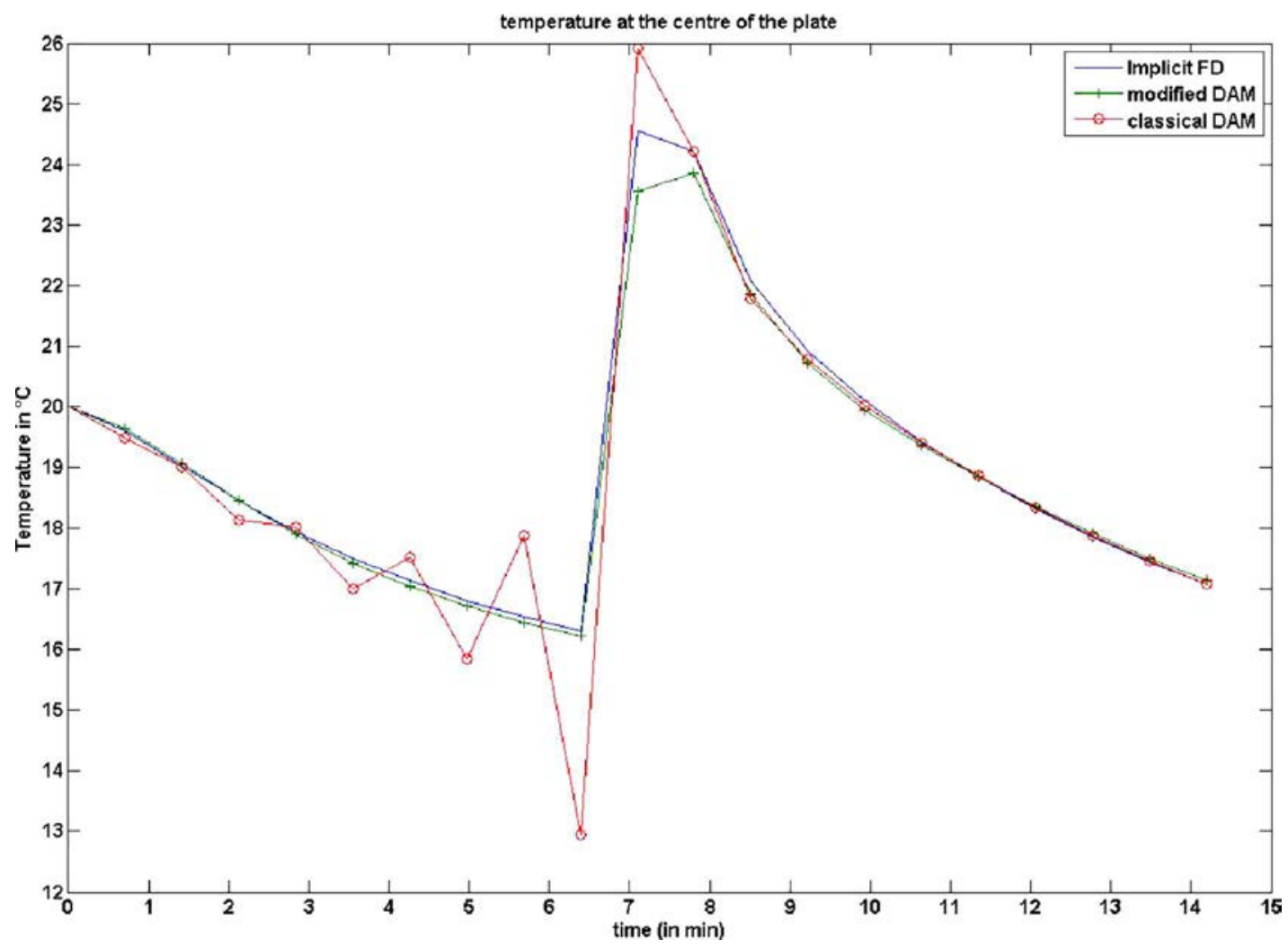

Figure 6. Temperature field against time on the plate for a moving heat source starting at middle time $t_{\text {start }}=7$ min $7 \mathrm{~s}$ at the middle of a flat steel plate, and going along the diagonal at a speed of $0.17 \mathrm{~mm} / \mathrm{s}$, for $n_{x}=n_{y}=n_{\mathrm{T}}=21, \mathrm{Bi}=1$, and $P_{\max }=10 \mathrm{MW} / \mathrm{m}^{3}$. 


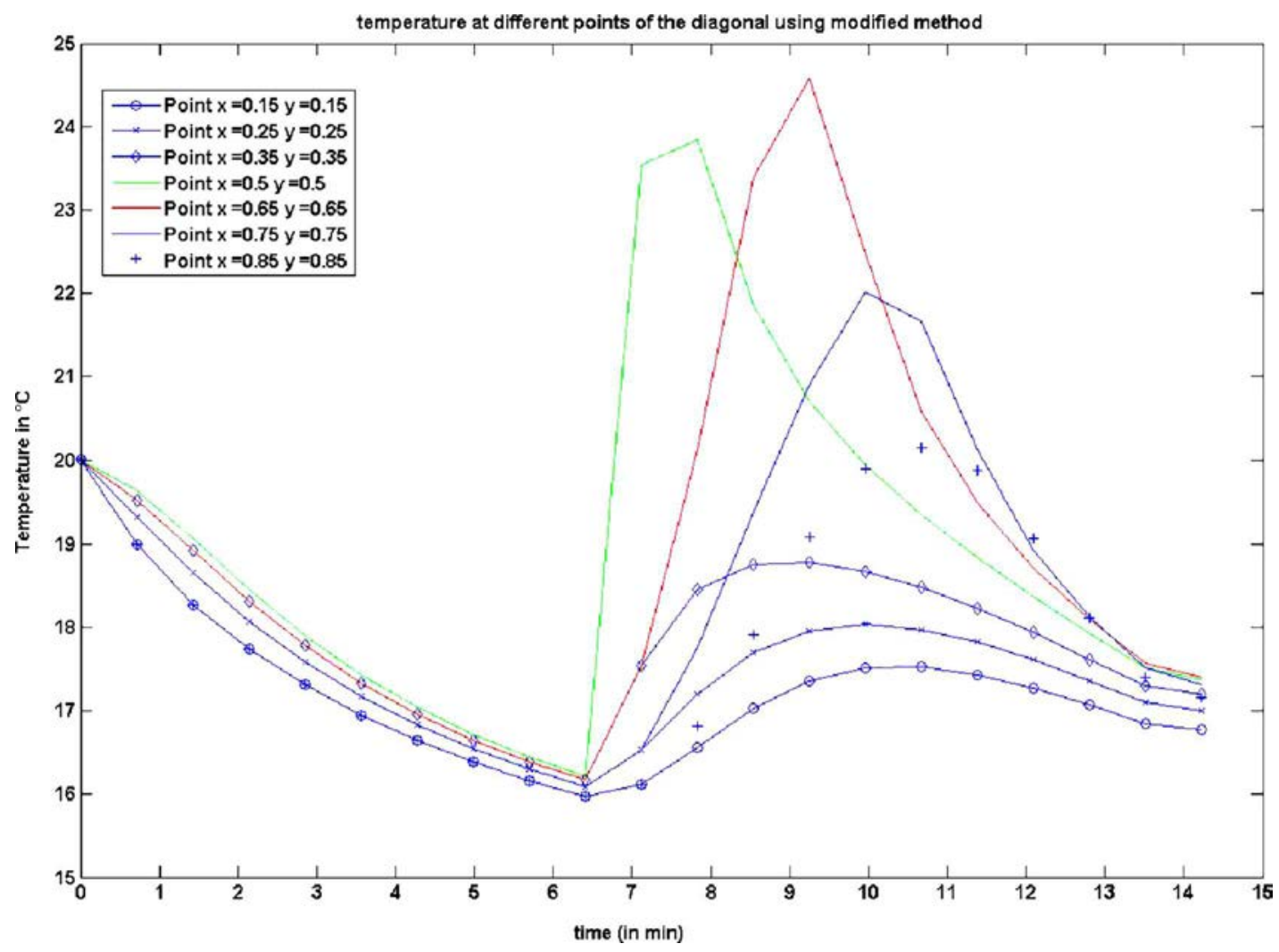

Figure 7. Temperature against time for a moving heat source along the diagonal of the plate and starting at middle time, for $n_{x}=n_{y}=n_{\tau}=21, \mathrm{Bi}=1$, and $P_{\max }=10 \mathrm{MW} / \mathrm{m}^{3}$ (using modified method).

The source applied first in the midplate at the physical starting time of $7 \mathrm{~min} 7 \mathrm{~s}$ moves at a speed of $0.17 \mathrm{~mm} / \mathrm{s}^{1}$ along the diagonal. The Biot number and maximum power $P_{\max }$ were set to 1 and $10 \mathrm{MW} / \mathrm{m}^{3}$, respectively. This problem has been solved by our space-time meshless method with the classical and the backward weight functions and with the implicit in finite difference time method. We first present in Figure 6 the time variation of temperature at the center of the plate. One can see that the "backward" weight function leads to smooth results comparable in accuracy to those given by the finite difference method. On the other hand, the classical weight function implies the failure of the meshless method by introducing numerical oscillations. Temperature evolutions with time at some points along the diagonal obtained with the backward weight function are finally given in Figure 7. Once again, one notices that the profiles are smooth and free from any oscillation.

\section{Conclusion}

A fully space-time meshless method to solve two-dimensional in space heat conduction problems with strong source discontinuities has been presented. This method does not require any secondary finite difference time-stepping procedure. The classical and backward weight functions were used. It has been shown that when there is no source term or when the source term is not discontinuous in time, both weight functions lead to smooth and accurate results. However, in the presence of sharp source discontinuities, the classical weight function leads to inaccurate results with spurious numerical oscillations, while the backward weight function appears to be accurate and oscillation free. 


\section{References}

[1] B. Nayroles, G. Touzot, and P. Villon, Generalizing the Finite Element Method: Diffuse Approximation and Diffuse Elements, Comput. Mech., vol. 10, pp. 307-318, 1992.

[2] T. Belytschko, Y. Y. Lu, and L. Gu, Element-Free Galerkin Methods, Int. J. Numer. Methods Eng., vol. 37, pp. 229256, 1994.

[3] H. Xiang and X. Zhang, Variational Multiscale Element-Free Galerkin Method and Precise Time Step Integration Method for Convection-Diffusion Problems, Numer. Heat Transfer, Part A, vol. 67, pp. 210-223, 2015.

[4] X. Zhang and P. Zhang, Heterogeneous Heat Conduction Problems by an Improved Element-Free Galerkin Method, Numer. Heat Transfer, Part B, vol. 65, pp. 359-375, 2014.

[5] S. N. Atluri and T. Zhu, A New Meshless Local Petrov-Galerkin (MLPG) Approach, Comput. Mech., vol. 22, pp. 117-127, 1998.

[6] L. Gao, K. Liu, and Y. Liu, A Meshless Method for Stress-Wave Propagation in Anisotropic and Cracked Media, Int. J. Eng. Sci., vol. 45, pp. 601-616, 2007.

[7] E. Shivanian, Meshless Local Petrov-Galerkin (MLPG) Method for Three-Dimensional Nonlinear Wave Equations Via Moving Least Squares Approximation, Eng. Anal. Boundary Elem., vol. 50, pp 249-257, 2015.

[8] X. T. Pham, Two-Dimensional Rosenthal Moving Heat Source Analysis Using the Meshless Element Free Galerkin Method, Numer. Heat Transfer, Part A, vol. 63, pp. 807-823, 2013.

[9] H. Huang and F. Costanzo, On the Use of Space-Time Finite Elements in the Solution of Elasto-Dynamic Problems with Strain Discontinuities, Comput. Methods Appl. Mech. Eng., vol. 191, pp. 5315-5343, 2002.

[10] J. Chessa and T. Belytschko, A Local Space-Time Discontinuous Finite Element Method, Comput. Methods Appl. Mech. Eng., vol. 195, pp. 1325-1343, 2006.

[11] J. Réthoré, A. Gravouil, and A. Combescure, A Combined Space-Time Extended Finite Element Method, Int. J. Numer. Methods Eng., vol. 64, pp. 260-284, 2005.

[12] S. Wang, J. Goldak, J. Zhou, S. Tchernov, and D. Downey, Simulation on the Thermal Cycle of a Welding Process by Space-Time Convection-Diffusion Finite Element Analysis, Int. J. Thermal Sci., vol. 48, pp. 936-947, 2009.

[13] D. K. Khalmanova and F. Costanzo, A Space-Time Discontinuous Galerkin Finite Element Method for Fully Coupled Linear Thermo-Elasto-Dynamic Problems with Strain and Heat Flux Discontinuities, Comput. Methods Appl. Mech. Eng., vol. 197, pp. 1323-1342, 2008.

[14] A. Legay, A. Zilian, and C. Janssen, A Rheological Interface Model And Its Space-Time Finite Element Formulation For Fluid-Structure Interaction, Int. J. Numer. Methods Eng., vol. 86, pp. 667-687, 2011.

[15] A. Zilian and A. Legay, The Enriched Space-Time Finite Element Method (EST) for Simultaneous Solution of Fluid-Structure Interaction, Int. J. Numer. Methods Eng., vol. 75, pp. 305-334, 2008.

[16] Z. K. Seymena, H. Yücel, and B. Karasözen, Distributed Optimal Control of Time-Dependent Diffusion-Convection-Reaction Equations Using Space-Time Discretization, J. Comput. Appl. Math., vol. 261, pp 146-157, 2014.

[17] M. Dumbser, O. Zanotti, A. Hidalgo, and D. S. Balsara, ADER-WENO Finite Volume Schemes with Space-Time Adaptive Mesh Refinement, J. Comput. Phys., vol. 248, pp. 257-286, 2013.

[18] F. Collino, T. Fouquet, and P. Joly, Conservative Space-Time Mesh Refinement Methods for the FDTD Solution of a Maxwell's Equations, J. Comput. Phys., vol. 211, pp. 9-35, 2006.

[19] V. Kostin, V. Lisitsa, G. Reshetova, and V. Tcheverda, Local Time-Space Mesh Refinement for Simulation of Elastic Wave Propagation in Multi-Scale Media, J. Comput. Phys., vol. 281, pp. 669-689, 2014.

[20] N. Netuzhylov and A. Zilian, Space-Time Meshfree Collocation Method: Methodology and Application to InitialBoundary Value Problems, Int. J. Numer. Methods Eng., vol. 80, pp. 355-380, 2009.

[21] C.-A. Wang, H. Sadat, and J.-Y. Tan, Meshless Method for Solving Transient Radiative and Conductive Heat Transfer in Two-Dimensional Complex Geometries, Numer. Heat Transfer, Part B, vol. 65, pp. 518-536, 2014.

[22] T. Sophy, A. Da Silva, and A. Kribèche, A Space-Time Meshless Method that Removes Numerical Oscillations When Solving PDEs with High Discontinuities, Numer. Heat Transfer, Part B, vol. 62, pp. 50-70, 2012.

[23] T. Sophy, H. Sadat, and C. Prax, A Meshless Formulation for Three-Dimensional Laminar Natural Convection, Numer. Heat Transfer, Part B, vol. 41, pp. 433-445, 2002.

[24] K. Reuther, B. Sarler, and M. Rettenmayr, Solving Diffusion Problems on an Unstructured, Amorphous Grid by a Meshless Method, Int. J. Thermal Sci., vol. 55, pp. 16-22, 2012.

[25] J. Crank, The Mathematics of Diffusion, 2nd ed., reprint, Clarendon Press, Oxford, 1979. 